ARTIGO ORIGINAL

\title{
Avaliação de ingredientes ativos para controle de plantas daninhas em plantio clonal de eucalipto no sistema de remonta e sem remonta
}

\author{
Assessment of active ingredients for weed control in eucalyptus clonal \\ plants
}

Melrian Schetz ${ }^{1}$ (D), Mariane Bueno de Camargo ${ }^{2}$ (D), Eduardo Henrique Rezende ${ }^{1}$ (D), Diego Tyszka Martinez ${ }^{1}$ (i), Nilton José Sousa ${ }^{1}$ (B)

'Universidade Federal do Paraná - UFPR. Curitiba, PR, Brasil

${ }^{2}$ Klabin S.A. Telêmaco Borba, PR, Brasil

Como citar: Schetz, M., Camargo, M. B., Rezende, E. H., Martinez, D. T., Sousa, N. J. (2021). Avaliação de ingredientes ativos para controle de plantas daninhas em plantio clonal de eucalipto no sistema de remonta e sem remonta. Scientia Forestalis, 49(129), e3395. https://doi.org/10.18671/scifor.v49n129.15

\begin{abstract}
Resumo
O objetivo do estudo foi determinar a eficiência de ingredientes ativos sobre o controle de plantas daninhas em plantio clonal de híbrido de Eucalyptus grandis x Eucalyptus urophylla, com e sem remonta (reaplicação) de produtos com ação de pré-emergência, bem como avaliar a intoxicação visual sobre as mudas submetidas a esta operação. A pesquisa foi realizada em Telêmaco Borba - PR, entre março e dezembro de 2018, por meio da combinação de herbicidas, com e sem remonta. Os produtos testados foram: glyphosate, isoxaflutole, flumioxazina, indaziflam, carfentrazona-etílica, saflufenacil e clomazona. As avaliações de intoxicação visual foram realizadas 10 dias após o plantio e 10 dias após a realização da primeira remonta de pré-emergentes. As avaliações de eficiência foram realizadas em intervalos de 40 dias após cada época da primeira aplicação (DAA) até o fechamento das copas do eucalipto. A primeira remonta foi realizada 120 DAA. Os herbicidas não causaram intoxicação visual no eucalipto. As análises estatísticas revelaram diferença significativa entre os tratamentos de controle de plantas daninhas. Conclui-se que as misturas de ingredientes ativos foram eficientes e a remonta, aos 120 DAA, permitiu bom controle até o fim do estudo. Glyphosate + isoxaflutole + remonta apresentou excelente eficiência na linha e entrelinha aos 240 dias, com a utilização de apenas uma operação de pré-emergente e pósemergente.
\end{abstract}

Palavras-chave: Controle químico; Intoxicação visual; Herbicida.

\begin{abstract}
The objective of this study was to determine the efficiency of active ingredients on the control of weeds in clonal plantations of hybrid Eucalyptus grandis $x$ Eucalyptus urophylla, with and without reapplication of pre-emergent herbicide and trace the visual phytotoxic effects on the seedlings. The research was carried out at Telêmaco Borba - PR, in the period from March to December 2018, combinations of herbicide management, with and without reapplication of pre-emergent herbicide, were evaluated: glyphosate, isoxaflutole, flumioxazine, indaziflam, carfentrazone-ethylic, saflufenacil and clomazone. Evaluations of visual phytotoxicity were performed 10 days after planting and 10 days after the first reapplication of preemergent herbicide on selected allotments. Efficiency evaluations were performed every 40 days after application (DAA) until canopy closure. The first reapplication of pre-emergent herbicide was done 120 DAA. The tested pre-emergent herbicides did not cause visual phytotoxicity in the eucalyptus clones. Statistical analysis revealed a significant difference between treatments. The study concluded that the mix of active ingredients was efficient for the control of weeds present in the area and the reapplication
\end{abstract}

Fonte de financiamento: Nenhuma.

Conflito de interesse: Nada a declarar.

Autor correspondente: melschetz@gmail.com

Recebido: 29 maio 2019.

Aceito: 29 maio 2020

Editor: Paulo Henrique Müller Silva.

(c) (†) Este é um artigo publicado em acesso aberto (Open Access) sob a licença Creative Commons Attribution, que permite uso, distribuição e reprodução em qualquer meio, sem restrições desde que o trabalho original seja corretamente citado. 
of pre-emergent herbicide at 120 DAA, allowed for an efficient weed control until the end of the experiment. Glyphosate + isoxaflutole with reapplication of pre-emergent presented excellent weed control at 240 days in the lines and between lines, using only one pre-emergent and one post-emergent herbicide.

Keywords: Chemical control; Visual intoxication; Herbicide.

\section{INTRODUÇÃO}

O Brasil possui 7,84 milhões de hectares de florestas plantadas, sendo que 5,8 milhões de hectares são plantios do gênero Eucalyptus (Indústria Brasileira de Árvores, 2018). O sucesso do gênero no Brasil deve-se aos elevados níveis de produtividade das florestas plantadas, aos avanços no melhoramento genético e melhoria dos processos de silvicultura.

Entre os vários processos silviculturais, o manejo adequado de plantas daninhas é um dos fatores que influenciam diretamente na produtividade tornando um desafio para os silvicultores, pois depende do grau de infestação e espécie das plantas daninhas, tipo de solo, condições climáticas, entre outros.

As práticas silviculturais de controle de plantas daninhas representam uma parcela significativa nos custos de implantação das florestas, em torno de 18 a 22\%, distribuídos entre insumo e serviços (Teixeira et al., 2015). Com o aumento dos preços, a automação das operações e o uso de moléculas mais modernas e eficientes (menores doses, maior efeito residual, aplicação em pré-emergência) mostram-se como alternativas promissoras para manutenção da qualidade da floresta e otimização dos custos (Teixeira et al., 2015).

A diversidade de herbicidas permite que seja realizado o melhor controle das diversas espécies de plantas daninhas presentes em uma área. O uso inadequado pode auxiliar na seleção de plantas cada vez mais tolerantes ou resistentes. O efeito dos diferentes herbicidas sobre os diferentes materiais genéticos ainda é pouco explorado na silvicultura.

Porém no Brasil são poucos os herbicidas registrados para a cultura do eucalipto quando comparado com outros países que também têm grandes áreas de eucalipto plantadas (Pereira \& Alves, 2015). No Brasil são registrados para o setor florestal um pequeno número de moléculas quando comparado ao setor agrícola, concentrando o uso principalmente em: glufosinate-ammonium, carfentrazona-etílica, flumioxazina, glyphosate, isoxaflutole, oxyfluorfen, sulfentrazone, saflufenacil, imazapyr, indaziflam, clomazone, haloxifope, triclopir e fluroxipir (Brasil, 2019).

Os sistemas de manejo das organizações certificadas pelo FSC (Forest Stewardship Council) devem promover o desenvolvimento e a adoção de métodos ambientalmente adequados de controle de pragas e doenças, e esforçarem-se para minimizar o uso de agrotóxicos. Assim devem demonstrar comprometimento em otimizar o uso de agrotóxicos, identificando riscos e analisando alternativas químicas e não-químicas de controle de pragas e doenças.

Diante deste contexto, a escolha consciente de um sistema de controle químico deve levar em conta a eficiência do herbicida ou das misturas de produtos, ingredientes ativos menos tóxicos ao meio ambiente e que não gerem danos na cultura de interesse, como intoxicação visual e redução do crescimento, assim estabelecendo um manejo específico para cada condição de campo encontrada.

Com isso, o objetivo desse estudo foi determinar a eficiência de mesclas de ingredientes ativos sobre o controle de plantas daninhas em plantio clonal de Eucalyptus grandis $\mathrm{x}$ Eucalyptus urophylla, com e sem remonta de pré-emergente, e avaliar a intoxicação visual das moléculas sobre as mudas.

\section{MATERIAL E MÉTODOS}

O estudo foi realizado em condições de campo no município de Telêmaco Borba - Paraná, situado nas coordenadas $24^{\circ} 11^{\prime} 28^{\prime \prime} \mathrm{S}$ de latitude e $50^{\circ} 32^{\prime} 23^{\prime \prime} \mathrm{W}$ de longitude, e altitude de $823 \mathrm{~m}$.

Conforme a classificação de Köppen, a área encontra-se em uma região de transição climática entre $\mathrm{Cfa}$ e $\mathrm{Cfb}$, predominante subtropical transicional para o temperado, úmido, 
mesotérmico, sem estação seca definida. O verão é quente com concentração de chuvas, e, no inverno, baixa frequência de geadas.

O solo da área experimental foi classificado como Latossolo Vermelho de textura argilosa e a região experimental possui um histórico de precipitação média mensal de $130 \mathrm{~mm}$. Durante o período do experimento as chuvas ocorreram de acordo com a Figura 1.

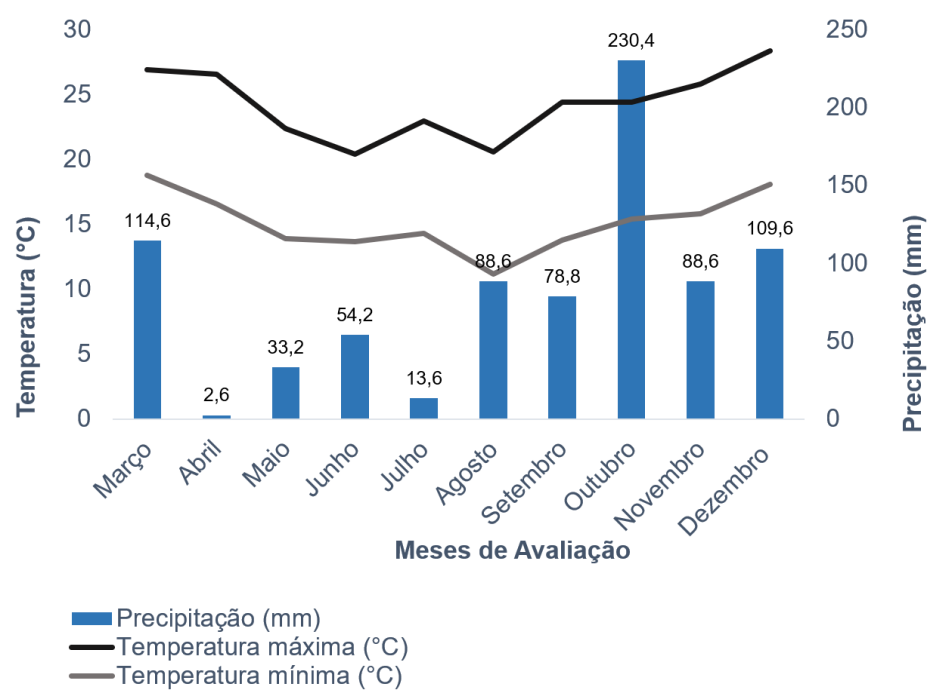

Figura 1: Dados climáticos da área experimental no período estudado.

Este experimento foi instalado em uma área de reforma de Eucalyptus com presença de resíduos da colheita anterior. O preparo do solo foi realizado em 06 de fevereiro de 2018 com uma subsolagem na linha de plantio potencializando as condições do solo para o crescimento inicial das mudas.

Após esta etapa, realizou-se o controle de formigas cortadeiras pré-plantio, em 09 de março, de maneira sistemática utilizando $4 \mathrm{~kg} / \mathrm{ha}$ de iscas granuladas. O plantio foi realizado no dia 20 de março de 2018 e esse mesmo controle de formigas foi realizado cinco meses após o plantio.

A área experimental possui 4,6 hectares, sendo que cada parcela amostral possui $420 \mathrm{~m}^{2}$ $(16,5 \mathrm{~m} \times 25,4 \mathrm{~m})$ contendo 5 linhas de plantio e 14 plantas por linha. As linhas externas de cada lado e as plantas da extremidade de cada linha constituíram as bordaduras.

Os tratamentos para a dessecação total da cobertura infestante na área, pré-plantio, foram instalados em 14 de março de 2018. O delineamento experimental utilizado foi blocos casualizados (DBC) com 27 tratamentos e 4 repetições cada. A comunidade de plantas presentes na área experimental se caracterizava predominantemente pelo gênero Urochloa sp., popularmente chamada de braquiária, e em menor proporção pelas espécies: Sida rhombifolia (guanxuma), Erechtites valerianaefolia (capiçova), Phytolacca thyrsiflora (caruru), Solanum granuloso-leprosum (fumo-bravo), Asclepias curassavica (paina-de-sapo).

O espaçamento utilizado para o plantio foi de 1,8 $\mathrm{m}$ × 3,3 m. As mudas foram produzidas no próprio viveiro da empresa e o plantio clonal de $E$. urophylla $x$ E. grandis foi realizado cinco dias após aplicação dos herbicidas.

Aos 45 dias após o plantio foi realizada uma adubação de base em todas as plantas. Conforme análise prévia de solo, utilizou-se 150 gramas de adubo por muda composto de NPK na formulação 04-42-06 aplicado manualmente em covetas laterais e aos 100 dias após o plantio foi realizada uma adubação de cobertura com 150 gramas da formulação de 10-05-30 ao redor da planta.

Foram testados diferentes ingredientes ativos, mecanismos de ação e classificações de acordo com a época de aplicação, ou seja, pré-emergência e pós-emergência, todos os herbicidas com registro para o gênero Eucalyptus. A aplicação dos tratamentos foi realizada com o uso de pulverizador costal manual de capacidade de 20 litros e bicos de pulverização Teejet Turbo Induction (TTI). O volume de calda preparada foi de $130 \mathrm{~L} / \mathrm{ha}$. 
A determinação da dose testada foi realizada a partir da dosagem recomendada pela bula dos produtos comerciais (Brasil, 2019) para a textura de solo argilosa (Tabela 1), sendo elas:

Tabela 1: Características químicas e físicas dos ingredientes ativos testados.

\begin{tabular}{cccc}
\hline Ingrediente ativo & Concentração & Dose de produto & Modalidade \\
\hline Glyphosate & $500 \mathrm{~g} / \mathrm{L}$ & $2800 \mathrm{~mL} / \mathrm{ha}$ & Pós-emergente \\
Isoxaflutole & $750 \mathrm{~g} / \mathrm{kg}$ & $200 \mathrm{~g} / \mathrm{ha}$ & Pré-emergente \\
Flumioxazina & $500 \mathrm{~g} / \mathrm{kg}$ & $180 \mathrm{~g} / \mathrm{ha}$ & Pré-emergente \\
Indaziflam & $500 \mathrm{~g} / \mathrm{L}$ & $150 \mathrm{~mL} / \mathrm{ha}$ & Pré-emergente \\
Carfentrazona-etílica & $400 \mathrm{~g} / \mathrm{L}$ & $75 \mathrm{~mL} / \mathrm{ha}$ & Pós-emergente \\
Saflufenacil & $700 \mathrm{~g} / \mathrm{kg}$ & $75 \mathrm{~mL} / \mathrm{ha}$ & Pós-emergente \\
Clomazona & $360 \mathrm{~g} / \mathrm{L}$ & $3000 \mathrm{~mL} / \mathrm{ha}$ & Pré-emergente \\
\hline
\end{tabular}

Com exceção do Tratamento 1 que não recebeu controle, todos os demais receberam a aplicação de glyphosate em área total (Tabela 2). Os tratamentos testados foram:

Tabela 2: Interação de ingredientes ativos testados por tratamento.

\begin{tabular}{|c|c|c|c|c|}
\hline Local & Tratamento & & gredientes Ati & \\
\hline \multirow{7}{*}{ Área Total } & $\mathrm{T} 1$ & Testemunha & \multirow[b]{2}{*}{ Isoxaflutol } & \\
\hline & $\mathrm{T} 2$ & Glyphosate & & \\
\hline & $\mathrm{T} 3$ * & Glyphosate & Isoxaflutol & \\
\hline & $\mathrm{T} 4$ & Glyphosate & Isoxaflutol & Carfentrazona-etílica \\
\hline & $\mathrm{T} 5$ * & Glyphosate & Isoxaflutol & Carfentrazona-etílica \\
\hline & T6 & Glyphosate & Isoxaflutol & Saflufenacil \\
\hline & $\mathrm{T} 7$ * & Glyphosate & Isoxaflutol & Saflufenacil \\
\hline Linha & \multirow{2}{*}{ T8 } & Glyphosate & Isoxaflutol & \\
\hline Entrelinha & & Glyphosate & Indaziflam & \\
\hline Linha & \multirow{2}{*}{ T9 * } & Glyphosate & Isoxaflutol & \\
\hline Entrelinha & & Glyphosate & Indaziflam & \\
\hline Linha & \multirow{2}{*}{$\mathrm{T} 10$} & Glyphosate & Isoxaflutol & Carfentrazona-etílica \\
\hline Entrelinha & & Glyphosate & Indaziflam & Carfentrazona-etílica \\
\hline Linha & \multirow{2}{*}{$\mathrm{T} 11$ * } & Glyphosate & Isoxaflutol & Carfentrazona-etílica \\
\hline Entrelinha & & Glyphosate & Indaziflam & Carfentrazona-etílica \\
\hline Linha & \multirow{2}{*}{$\mathrm{T} 12$} & Glyphosate & Isoxaflutol & Saflufenacil \\
\hline Entrelinha & & Glyphosate & Indaziflam & Saflufenacil \\
\hline Linha & \multirow{2}{*}{$\mathrm{T} 13$ * } & Glyphosate & Isoxaflutol & Saflufenacil \\
\hline Entrelinha & & Glyphosate & Indaziflam & Saflufenacil \\
\hline \multirow{6}{*}{ Área Total } & T14 & Glyphosate & Flumioxazina & \\
\hline & $\mathrm{T} 15$ * & Glyphosate & Flumioxazina & \\
\hline & T16 & Glyphosate & Flumioxazina & Carfentrazona-etílica \\
\hline & $\mathrm{T} 17$ * & Glyphosate & Flumioxazina & Carfentrazona-etílica \\
\hline & T18 & Glyphosate & Flumioxazina & Saflufenacil \\
\hline & $\mathrm{T} 19$ * & Glyphosate & Flumioxazina & Saflufenacil \\
\hline Linha & \multirow{2}{*}{ T20 } & Glyphosate & Flumioxazina & \\
\hline Entrelinha & & Glyphosate & Indaziflam & \\
\hline Linha & $\mathrm{T} 21$ * & Glyphosate & Flumioxazina & \\
\hline
\end{tabular}


Tabela 2: Continuação...

\begin{tabular}{|c|c|c|c|c|}
\hline Local & Tratamento & \multicolumn{3}{|c|}{ Ingredientes Ativos } \\
\hline Entrelinha & & Glyphosate & Indaziflam & \\
\hline Linha & \multirow{2}{*}{$\mathrm{T} 22$} & Glyphosate & Flumioxazina & Carfentrazona-etílica \\
\hline Entrelinha & & Glyphosate & Indaziflam & Carfentrazona-etílica \\
\hline Linha & \multirow{2}{*}{$\mathrm{T} 23$ * } & Glyphosate & Flumioxazina & Carfentrazona-etílica \\
\hline Entrelinha & & Glyphosate & Indaziflam & Carfentrazona-etílica \\
\hline Linha & \multirow{2}{*}{$\mathrm{T} 24$} & Glyphosate & Flumioxazina & Saflufenacil \\
\hline Entrelinha & & Glyphosate & Indaziflam & Saflufenacil \\
\hline Linha & \multirow{2}{*}{$\mathrm{T} 25$ * } & Glyphosate & Flumioxazina & Saflufenacil \\
\hline Entrelinha & & Glyphosate & Indaziflam & Saflufenacil \\
\hline Área Total & T26 & Glyphosate & Clomazona & Carfentrazona-etílica \\
\hline Área Total & $\mathrm{T} 27$ * & Glyphosate & Isoxaflutol & Flumioxazina Carfentrazona-etílica \\
\hline
\end{tabular}

*Tratamentos com reaplicação de pré-emergente aos 120 DAA.

Após a dessecação, monitorou-se a necessidade de realizar-se a reaplicação de préemergentes em área total, popularmente chamada de remonta. Essa atividade foi realizada aos 120 dias após a primeira aplicação, este intervalo de reaplicação foi de acordo com monitoramento prévio da área que ocorreu a cada 40 dias.

Foram avaliadas intoxicação visual dos ingredientes ativos no clone eucalipto e eficiência dos ingredientes ativos no controle das plantas daninhas.

A intoxicação visual foi realizada 10 dias após o plantio das mudas e 10 dias após a reaplicação do tratamento de ação de pré emergência (remonta) nas mudas centrais da parcela. Para a determinação dos efeitos tóxicos dos tratamentos testados, a avaliação foi realizada de forma visual conforme o método descrito pela Sociedade Brasileira de Ciência das Plantas Daninhas (Sbcpd, 1995), que possui escala de notas, conforme descrição na Tabela 3.

Tabela 3: Escala de notas utilizada para avaliação visual de intoxicação das plantas por tratamento.

\begin{tabular}{|c|c|c|}
\hline Conceito & Notas & Observação \\
\hline Muito leve & $0-5$ & $\begin{array}{l}\text { Sintomas fracos ou pouco evidentes. Nota zero quando não se } \\
\text { observam quaisquer alterações na cultura. }\end{array}$ \\
\hline Leve & $6-10$ & Sintomas nítidos, de baixa intensidade. \\
\hline Moderada & $11-20$ & Sintomas nítidos, mais intensos que na classe anterior. \\
\hline Aceitável & $21-35$ & Sintomas pronunciados, porém totalmente tolerados pela cultura. \\
\hline Preocupante & $36-45$ & $\begin{array}{c}\text { Sintomas mais drásticos que na categoria anterior, mas ainda } \\
\text { passíveis de recuperação, e sem expectativas de redução no } \\
\text { rendimento econômico. }\end{array}$ \\
\hline Alta & $46-60$ & $\begin{array}{c}\text { Danos irreversíveis, com previsão de redução no rendimento } \\
\text { econômico. }\end{array}$ \\
\hline Muito alta & $61-100$ & $\begin{array}{c}\text { Danos irreversíveis muito severos, com previsão de redução drástica } \\
\text { no rendimento econômico. Nota } 100 \text { para morte de toda a cultura }\end{array}$ \\
\hline
\end{tabular}

Fonte: Sbcpd (1995).

Para avaliação da eficiência foram consideradas as 3 linhas centrais de cada parcela. Os efeitos do herbicida nas plantas daninhas foram avaliados pelo método qualitativo com observações visuais utilizando uma escala percentual de notas variando entre 0 (zero) e 100 (cem), onde 0 implica ausência de controle e 100, o controle total das plantas daninhas. Utilizou-se a metodologia proposta pela Asociación Latinoamericana de Malezas (Asociation Latinoamericana de Malezas, 1974), que atribui nota de controle, bem como seu conceito para cada classe de porcentagem estabelecida (Tabela 4). 
Tabela 4: Escala de notas utilizada para avaliação do controle de plantas daninhas de acordo com a eficiência nos tratamentos.

\begin{tabular}{ccc}
\hline Notas & Classe de porcentagem (\%) & Conceito de controle \\
\hline 1 & $0-40$ & Nenhum/pobre \\
2 & $41-60$ & Regular \\
3 & $61-70$ & Suficiente \\
4 & $71-80$ & Bom \\
5 & $81-90$ & Muito bom \\
6 & $91-100$ & Excelente
\end{tabular}

Fonte: Asociation Latinoamericana de Malezas (1974).

As avaliações visuais de controle das plantas daninhas pelos herbicidas foram realizadas aos 40,80,120,160, 200 e 240 DAA. Os dados obtidos foram submetidos à análise estatística através do software "Statistical Analysis System" (SAS Learning Edition, 2003). Os resultados foram testados conforme Coimbra et al. (2004). As variáveis: eficiência do tratamento (x) no controle das plantas daninhas na linha de plantio e na entrelinha foram submetidos ao teste de Bartlett para testar a homogeneidade dos dados. Os efeitos dos tratamentos: Herbicida (combinações de herbicidas) e Época (Dias Após Aplicação - DAA) foram testados por meio de Análise de Variância (ANOVA). Utilizou-se o modelo para parcelas subdivididas, em que as combinações de herbicidas foram testadas por meio do Erro a (parcelas) e DAA por meio do Erro $b$ (subparcelas). Vale salientar que o fator herbicida foi comparado por meio do Teste de Tukey e o fator DAA por análise de regressão, sendo testados os graus dos polinômios por meio do Teste F, com 95\% de probabilidade.

\section{RESULTADOS E DISCUSSÃO}

\section{Produtos de Ação Pós-Emergência}

A avaliação de intoxicação visual, realizada 10 dias após o plantio, não detectou sintomas nas mudas de eucaliptos. Esse fato é justificado pelas características dos produtos de pós-emergência testados, bem como por terem sido aplicado ao que antecedeu aos plantios das mudas.

Os herbicidas com princípios ativos carfentrazona-etilica e saflufenacil não possuem persistência no solo e precisam ser aplicados no alvo biológico (plantas daninhas) para atuarem, pois são classificados como herbicidas de contato. O plantio do eucalipto foi realizado cinco dias após a aplicação desses herbicidas, por isso não foram observados sintomas de intoxicação visual.

A molécula de glyphosate testada também possui ação de pós emergência e não foi aplicada sobre as mudas de eucalipto. Esse ingrediente ativo possui ação sistêmica, o que auxilia na translocação dos produtos de ação de contato testados fazendo com que atuem melhor no controle da planta alvo. Esta molécula não possui ação de pré-emergência por ser fortemente adsorvida e degradada quando em contato com o solo exposto.

Aos 120 DAA, na entrelinha das parcelas com remonta, foram aplicadas glyphosate (1400 g i.a.ha-1) para controlar as plantas que já estavam em desenvolvimento. Os sintomas visuais de intoxicação por glyphosate são cloroses e necroses foliares, sendo que as plantas com maior intensidade de intoxicação apresentavam clorose e morte dos ápices caulinares (Costa et al., 2012).

\section{Produtos de Ação Pré-Emergência}

$O$ indaziflam possui residual prolongado que poderia afetar o desenvolvimento radicular das mudas de eucaliptos, por isso foi aplicado apenas na entrelinha (conforme recomendação descrita em bula).

Mesmo o clomazone considerado volátil (Brasil, 2019) não foram observados danos visuais que afetassem a produtividade das mudas de eucalipto. 
Os ingredientes ativos isoxaflutole e flumioxazina são herbicidas de ação sistêmica, em sua maior parte absorvidos pelas raízes (Brasil, 2019). No entanto, não foram observados efeitos de intoxicação visual e redução de crescimento do clone avaliado, tanto aos 10 dias após o plantio quanto na aplicação de remonta aos 120 DAA.

Sabe-se que sintomas de intoxicação nas plantas de eucalipto ocasionados pelo flumioxazina são caracterizados por cloroses nas folhas do terço inferior das plantas (Rezende, 2014). Tiburcio et al. (2012) não observaram sintomas de intoxicação nas folhas jovens do ápice caulinar bem como nas brotações emitidas pelo clone CRV1189 de Eucalyptus grandis quando submetidos a doses de flumioxazina $\left(75,100\right.$ e $125 \mathrm{~g}$ i.a.ha $\left.{ }^{-1}\right)$. No entanto, as plantas tratadas com flumioxazina (100 g i.a.ha-1 $)+$ sulfentrazone (500 g i.a.ha $\left.{ }^{-1}\right)$ foram mais intoxicadas até os 15 DAA $(71,25 \%)$.

Os tratamentos que receberam a remonta (120 dias após plantio) também não apresentaram sintomas de intoxicação visual quando avaliados 10 DAA. As plantas avaliadas estavam com altura média de $77 \mathrm{~cm}$ e diâmetro de colo médio de $15,6 \mathrm{~mm}$. Os tratamentos com flumioxazina foram aplicados com uma concentração maior (180 $\mathrm{g}$ i.a.ha- $\left.{ }^{-1}\right)$ que as testadas por Tiburcio et al. (2012). No entanto, o clone de E. urograndis também não apresentou sintomas de intoxicação. Ressalta-se a reaplicação, em jato dirigido, dos pré-emergentes, evitando-se, assim, a deriva dos produtos comerciais sobre as plantas de eucalipto.

Agostinetto et al. (2010) testaram diferentes doses de isoxaflutole, oxyfluorfen, bispyribac-sodium e sethoxydim + diclosulam em pós-emergência na cultura do eucalipto. Observaram que isoxaflutole (em doses de 37,5; 75; 112,5 e $150 \mathrm{~g}$ i.a ha ${ }^{-1}$ ) causou menor intoxicação para as espécies de E. saligna e E. globulus. Aos 28 DAA as plantas apresentavam intoxicação de $0,3 \%$. Nesta pesquisa, isoxaflutole mesmo em dose superior (200 g i.a.ha-1 $)$ não provocou sintomas de intoxicação sobre o clone de E. urograndis.

Os dois herbicidas reaplicados na linha de plantio possuem diferentes mecanismo de ação, um inibe a PROTOX (flumioxazina) e o outro a síntese de carotenoides (isoxaflutole) e não apresentaram sintomas de intoxicação no clone testado.

\section{Eficiência dos herbicidas}

Apesar do interesse em se obter a melhor combinação de ingredientes ativos para aplicação em área total, as avalições de eficiência de controle de plantas daninhas foram realizadas separadas. Avaliou-se eficiência na linha e na entrelinha de plantio devido ao uso do ingrediente ativo indaziflam (recomendado em bula para utilização apenas na entrelinha do plantio).

A análise de variância revelou diferença significativa para as variáveis de eficiência do tratamento na linha de plantio e entrelinha para os fatores Herbicida (combinações de herbicidas) e Época (Dias Após Aplicação - DAA), a análise dos dados também indicou interação significativa entre o fator herbicida e época de aplicação (Tabela 5).

Tabela 5: Análise de variância dos tratamentos, época e interação entre época e herbicida na linha e entrelinha de plantio.

\begin{tabular}{cccc}
\hline \multirow{2}{*}{ Fonte de Variação } & GL & \multicolumn{2}{c}{ Quadrado Médio (QM) } \\
\cline { 3 - 4 } & & LINHA & ENTRELINHA \\
\hline Bloco & 3 & $266,69^{* *}$ & $117,16^{* *}$ \\
Herbicida & 26 & $1044,4^{* *}$ & $1729,44^{* *}$ \\
Erro a (parcelas) & 78 & $109,14^{* *}$ & $144,46^{* *}$ \\
Época & 5 & $5431,86^{* *}$ & $9538,2 * *$ \\
Herbicida x Época & 130 & $173,8^{* *}$ & $144,47^{* *}$ \\
Erro b (subparcelas) & 360 & $25,93^{* *}$ & $25,35^{* *}$ \\
& $\mathrm{CV}$ & $5,98 \%$ & $6,04 \%$ \\
& $\mathrm{R}^{2}$ & 0,91 & 0,94 \\
\hline
\end{tabular}

**significativos a $1 \%$ 
Quando se analisou o fator época (DAA), entre os vários modelos de regressão testados, o que teve representatividade foi o modelo de regressão quadrática para todos os tratamentos.

Avaliou-se a eficiência das combinações de herbicidas ao longo do tempo e por quanto tempo esses ingredientes ativos conseguiram inibir a reinfestação das plantas daninhas, tanto na linha de plantio quanto na entrelinha.

Com exceção da testemunha (T1) que não recebeu controle de plantas daninhas, todos os tratamentos (T2 até T27) foram dessecados com, pelo menos, um herbicida pós-emergente a base de glyphosate acrescido de um pré-emergente visando o controle do banco de sementes e aumento do tempo de controle da área.

Os dados do Teste de Tukey a 5\% para a linha de plantio estão descritos na Tabela 6 e na Tabela 7 estão os resultados do Teste de Tukey a $5 \%$ da porcentagem de eficiência na entrelinha. Testou-se a porcentagem de eficiência nos períodos de 40, 80, 120, 160, 200 e 240 dias após a instalação do experimento.

Tabela 6: Teste de médias de eficiência de controle de plantas daninhas na linha de plantio por tratamento e época de avaliação.

\begin{tabular}{|c|c|c|c|c|c|c|c|c|c|c|c|c|}
\hline Tratamento & 40 & & 80 & & 12 & & 160 & & 200 & & 240 & \\
\hline 1 & 85,00 & b & 76,25 & c & 71,25 & c & 68,75 & d & 50,00 & $d$ & 5,00 & $f$ \\
\hline 2 & 96,50 & $a b$ & 93,75 & a & 90,00 & a & 90,00 & $b c$ & 88,75 & $a b$ & 82,50 & b \\
\hline 3 & 97,00 & $a b$ & 93,75 & $a$ & 87,50 & $a b$ & 100,00 & A & 95,00 & $a$ & 91,25 & $a$ \\
\hline 4 & 97,50 & $a$ & 88,04 & $a b$ & 81,37 & bc & 81,37 & $\mathrm{~cd}$ & 79,70 & bc & 71,37 & $\mathrm{~cd}$ \\
\hline 5 & 95,50 & $a b$ & 88,04 & $a b$ & 81,37 & bc & 96,37 & $a b$ & 93,04 & a & 83,04 & $a b$ \\
\hline 6 & 95,00 & $a b$ & 89,63 & $a b$ & 81,29 & bc & 86,29 & bc & 84,63 & b & 62,96 & de \\
\hline 7 & 95,00 & $a b$ & 90,63 & $a b$ & 82,96 & $a b c$ & 96,29 & $a b$ & 91,29 & $a b$ & 82,96 & $a b$ \\
\hline 8 & 96,50 & $a b$ & 88,75 & $a b$ & 83,75 & $a b$ & 85,00 & bc & 82,50 & bc & 66,25 & $d$ \\
\hline 9 & 95,00 & $a b$ & 90,00 & $a b$ & 83,75 & $a b$ & 96,25 & $a b$ & 92,50 & $a$ & 76,25 & bc \\
\hline 10 & 94,50 & $a b$ & 92,50 & $a b$ & 86,25 & $a b$ & 85,00 & bc & 81,25 & bc & 75,00 & c \\
\hline 11 & 93,75 & $a b$ & 86,25 & b & 76,25 & bc & 92,00 & b & 85,00 & b & 80,00 & bc \\
\hline 12 & 95,25 & $a b$ & 90,00 & $a b$ & 87,50 & $a b$ & 81,25 & $c d$ & 82,50 & bc & 73,75 & c \\
\hline 13 & 94,50 & $a b$ & 88,75 & $a b$ & 82,50 & bc & 98,75 & $a$ & 92,50 & $a$ & 88,75 & $a b$ \\
\hline 14 & 92,50 & $a b$ & 91,00 & $a b$ & 83,51 & $a b c$ & 78,51 & $\mathrm{~cd}$ & 78,51 & bc & 71,00 & $\mathrm{~cd}$ \\
\hline 15 & 95,00 & $a b$ & 93,51 & $a b$ & 78,51 & bc & 96,00 & $a b$ & 86,00 & $a b$ & 83,51 & $a b$ \\
\hline 16 & 95,00 & $a b$ & 90,00 & $a b$ & 78,75 & bc & 76,25 & $d$ & 73,75 & c & 56,25 & e \\
\hline 17 & 96,50 & $a b$ & 92,50 & $a b$ & 83,75 & $a b$ & 97,50 & $a$ & 92,50 & $a$ & 80,00 & bc \\
\hline 18 & 96,50 & $a b$ & 90,00 & $a b$ & 86,25 & $a b$ & 83,75 & c & 81,25 & bc & 62,50 & de \\
\hline 19 & 95,75 & $a b$ & 88,75 & $a b$ & 81,25 & bc & 96,25 & $a b$ & 88,75 & $a b$ & 87,50 & $a b$ \\
\hline 20 & 93,75 & $a b$ & 88,75 & $a b$ & 78,75 & bc & 77,50 & $c d$ & 73,75 & c & 58,75 & e \\
\hline 21 & 94,50 & $a b$ & 90,00 & $a b$ & 80,00 & $\mathrm{bc}$ & 97,50 & $a b$ & 92,50 & $a$ & 88,75 & $a b$ \\
\hline 22 & 94,50 & $a b$ & 86,45 & $a b$ & 83,11 & $a b c$ & 78,11 & $\mathrm{~cd}$ & 74,78 & c & 61,45 & de \\
\hline 23 & 93,75 & $a b$ & 87,50 & $a b$ & 81,25 & bc & 97,00 & $a b$ & 91,25 & $a b$ & 85,00 & $a b$ \\
\hline 24 & 92,50 & $a b$ & 85,00 & b & 78,75 & $\mathrm{bc}$ & 77,50 & $c d$ & 72,50 & c & 55,00 & e \\
\hline 25 & 90,00 & $b$ & 83,75 & $b c$ & 72,50 & c & 93,75 & $a b$ & 90,00 & $a b$ & 81,25 & bc \\
\hline 26 & 95,75 & $a b$ & 92,50 & $a b$ & 86,25 & $a b$ & 82,50 & $c d$ & 80,00 & bc & 71,25 & $\mathrm{~cd}$ \\
\hline 27 & 97,50 & a & 93,75 & a & 81,25 & bc & 95,00 & $a b$ & 90,00 & $a b$ & 78,75 & bc \\
\hline
\end{tabular}

Na coluna, médias seguidas de letras iguais não diferem entre si, pelo teste de Tukey (5\%). 
Tabela 7: Teste de médias de eficiência de controle de plantas daninhas na linha de plantio por tratamento e época de avaliação.

\begin{tabular}{|c|c|c|c|c|c|c|c|c|c|c|c|c|}
\hline \multirow{3}{*}{$\begin{array}{c}\text { Tratamento } \\
1\end{array}$} & \multicolumn{12}{|c|}{ Dias após instalação do experimento } \\
\hline & \multicolumn{2}{|l|}{40} & \multicolumn{2}{|c|}{80} & \multicolumn{2}{|c|}{120} & \multicolumn{2}{|c|}{160} & \multicolumn{2}{|c|}{200} & \multicolumn{2}{|c|}{240} \\
\hline & 71,25 & b & 65,00 & $\mathrm{C}$ & 62,50 & c & 58,75 & e & 45,00 & e & 0,00 & g \\
\hline 2 & 94,50 & a & 92,50 & a & 87,50 & $a b$ & 85,00 & bc & 80,00 & c & 68,75 & c \\
\hline 3 & 95,75 & a & 88,75 & $a b$ & 85,00 & $a b$ & 100,00 & a & 97,50 & a & 88,75 & $a b$ \\
\hline 4 & 95,50 & a & 89,96 & $a b$ & 79,96 & $b$ & 79,96 & $\mathrm{~cd}$ & 76,62 & c & 46,62 & ef \\
\hline 5 & 95,50 & $a$ & 88,29 & $a b$ & 81,62 & $a b$ & 99,96 & $a$ & 94,96 & $a b$ & 84,96 & $a b$ \\
\hline 6 & 94,50 & a & 84,64 & $b$ & 82,97 & $a b$ & 79,64 & $\mathrm{~cd}$ & 76,31 & C & 49,64 & ef \\
\hline 7 & 94,25 & $a$ & 89,64 & $a b$ & 79,63 & $b$ & 96,31 & $a$ & 87,97 & b & 81,31 & $b$ \\
\hline 8 & 94,00 & $a$ & 85,00 & $b$ & 82,50 & $a b$ & 80,00 & $\mathrm{~cd}$ & 78,75 & c & 56,25 & de \\
\hline 9 & 94,50 & $a$ & 87,50 & $a b$ & 82,50 & $a b$ & 98,75 & $a$ & 92,50 & $a b$ & 81,25 & $b$ \\
\hline 10 & 94,50 & $a$ & 90,00 & $a b$ & 87,50 & $a b$ & 85,00 & bc & 77,50 & c & 52,50 & e \\
\hline 11 & 93,75 & $a$ & 90,00 & $a b$ & 80,00 & $b$ & 97,50 & $a$ & 95,00 & $a b$ & 82,50 & b \\
\hline 12 & 94,00 & $a$ & 88,75 & $a b$ & 81,25 & $b$ & 78,75 & $\mathrm{~cd}$ & 77,50 & C & 61,25 & $d$ \\
\hline 13 & 96,50 & $a$ & 93,75 & $a$ & 85,00 & $a b$ & 100,00 & $a$ & 97,50 & $a$ & 87,50 & $\mathrm{db}$ \\
\hline 14 & 93,75 & $a$ & 93,11 & $a$ & 78,11 & b & 78,11 & $\mathrm{~cd}$ & 78,11 & c & 60,61 & de \\
\hline 15 & 91,25 & a & 88,11 & $a b$ & 73,11 & b & 100,00 & a & 90,61 & $a b$ & 70,61 & c \\
\hline 16 & 90,00 & a & 82,50 & $b$ & 76,25 & $b$ & 73,75 & $d$ & 68,75 & $d$ & 43,75 & $f$ \\
\hline 17 & 94,50 & $a$ & 88,75 & $a b$ & 83,75 & $a b$ & 100,00 & a & 96,25 & $a b$ & 76,25 & $b c$ \\
\hline 18 & 94,50 & $a$ & 90,00 & $a b$ & 85,00 & $a b$ & 80,00 & $\mathrm{~cd}$ & 77,50 & C & 47,50 & ef \\
\hline 19 & 95,00 & $a$ & 87,50 & $a b$ & 81,25 & b & 100,00 & a & 91,25 & $a b$ & 88,75 & $a b$ \\
\hline 20 & 92,50 & $a$ & 88,75 & $a b$ & 77,50 & b & 76,25 & $\mathrm{~cd}$ & 73,75 & $\mathrm{~cd}$ & 51,25 & e \\
\hline 21 & 95,25 & $a$ & 88,75 & $a b$ & 78,75 & $b$ & 100,00 & $a$ & 93,75 & $a b$ & 90,00 & a \\
\hline 22 & 93,75 & $a$ & 89,87 & $a b$ & 86,54 & $a b$ & 86,54 & bc & 84,87 & bc & 58,21 & de \\
\hline 23 & 94,50 & $a$ & 87,50 & $a b$ & 81,25 & $b$ & 100,00 & $a$ & 92,50 & $a b$ & 82,50 & b \\
\hline 24 & 94,50 & a & 91,25 & $a b$ & 81,25 & $b$ & 81,25 & c & 76,25 & $\mathrm{~cd}$ & 48,75 & ef \\
\hline 25 & 93,75 & a & 90,00 & $a b$ & 77,50 & $b$ & 95,00 & $a$ & 91,25 & $a b$ & 76,25 & bc \\
\hline 26 & 92,00 & $a$ & 88,75 & $a b$ & 85,00 & $a b$ & 80,00 & $\mathrm{~cd}$ & 77,50 & C & 50,00 & ef \\
\hline 27 & 95,00 & $a$ & 93,75 & $\mathrm{a}$ & 88,75 & $a$ & 90,00 & $b$ & 83,75 & $b c$ & 57,50 & de \\
\hline
\end{tabular}

Na coluna, médias seguidas de letras iguais não diferem entre si, pelo teste de Tukey (5\%).

Aos 40 DAA a eficiência dos ingredientes ativos não diferiu estatisticamente e a linha de plantio apresentou conceito excelente em praticamente todos os tratamentos (Tabela 6). Apesar desse conceito, a combinação com dois pré-emergentes glyphosate + isoxaflutole + flumioxazina + carfentrazona-etílica destacou-se com maior média (97,5\%).

No entanto, a combinação glyphosate + isoxaflutole + carfentrazona-etílica, com apenas um pré-emergente, também apresentou o mesmo resultado $(97,5 \%)$ com a vantagem do menor número de insumos utilizado para se obter o mesmo resultado, consequentemente gerando menor custo operacional.

Nessa mesma época, com exceção da testemunha (T1) todos os tratamentos tiveram excelente eficiência na entrelinha. Ou seja, o uso do glyphosate isolado ou acrescido de carfentrazona-etílica ou saflufenacil contribuíram para a dessecação da área.

Rezende (2014) testou diferentes pré-emergentes em área de implantação e reforma de eucalipto no estado de São Paulo, aos 40 DAA observando eficiência de controle superior a $96 \%$ para isoxaflutole e $95,62 \%$ para o flumioxazina. Apesar de regiões diferentes, esses índices de eficiência são próximos dos valores encontrados neste experimento aos 40 DAA.

$\mathrm{O}$ uso de um pré-emergente inicial na entrelinha contribuiu para maior período de controle de plantas daninhas, facilitando outras operações como adubação de base e 
cobertura e controle de formiga. Além disso, a manutenção da área limpa auxilia na diminuição dos riscos de acidentes à trabalhadores rurais e incêndios (Kofler \& Pandolfi, 2019).

Aos 80 DAA constatou-se visualmente uma porcentagem de reinfestação por plantas daninhas em todos os tratamentos, com diferença significativa para o tratamento testemunha (T1) (76,25\%). No tratamento T27, apesar de ter recebido a combinação de dois préemergentes de atuações diferentes, os dados obtidos indicaram a mesma eficiência de tratamentos com apenas um pré-emergente $(93,75 \%)$.

Rezende (2014) observou eficiência de controle de 81,62\% para o ingrediente ativo isoxaflutole e $78,87 \%$ para o flumioxazina na região noroeste do estado de São Paulo. Este experimento foi instalado em solo de textura argilosa, e no inverno, enquanto que o de Rezende (2014) em solos de textura arenosa, e no verão. Os resultados de Rezende 2014 podem ter sido diferentes devido às características de lixiviação diferentes dos solos e maiores volumes de precipitações ocorridas no verão destes produtos, o que infelizmente não se pode comparar ao trabalho de Rezende por não terem publicado esta informação.

Tiburcio et al. (2012) observaram em estudo de campo, no estado de Minas Gerais, que até os 45 DAA os tratamentos com isoxaflutole isolado apresentaram controle de monocotiledôneas quanto para dicotiledôneas abaixo de 75\%. Por outro lado, nesse experimento até 40 DAA todos os tratamentos estavam com controle excelente na entrelinha. Antes de realizar a remonta, os tratamentos T2, T3, T5, T6, T8, T9, T10, T13, T17, T18, T22, T26 e T27 apresentavam eficiência muito boa, a maior parte desses tratamentos receberam o ingrediente ativo isoxaflutole.

Dalazen et al. (2015) concluíram que os herbicidas glyphosate e saflufenacil possuem uma relação sinérgica principalmente no controle de plantas daninhas como a buva. Apesar da área testada não apresentar infestação de buva e sim predominantemente braquiária, essa combinação também apresentou efeitos positivos.

Durante as avaliações, o efeito residual dos herbicidas aplicados começou a diminuir, ao acompanhar mensalmente a eficiência dos 27 tratamentos, foi observado a necessidade de realizar a remonta aos 120 DAA, pois foi constatado o início da emergência das plantas daninhas na linha de plantio.

As aplicações de pré-emergentes (remonta) geralmente ocorrem em intervalos de cada 30 a 50 dias, conforme a região. Fazer a primeira remonta 120 dias após a primeira aplicação contribuiu para redução de atividade de controle (capina química) e insumos, consequentemente, redução de custo operacionais. Ressalta-se o fato do experimento ter sido conduzido no inverno (Figura 1), diminuindo naturalmente a infestação de plantas daninhas.

Ocorreu também um período de seca quando comparado com anos anteriores, no qual a precipitação média desse período era de $130 \mathrm{~mm}$ e no período estudado a maior precipitação mensal foi de 54,20 mm.

A reaplicação dos pré-emergentes (isoxaflutole e flumioxazina) na linha de plantio (tratamentos com remonta) garantiu eficiência de controle, verificado pelas avaliações aos 160, 200 e 240 dias (Tabela 6).

Aos 240 DAA, os melhores tratamentos foram T3, T5, T7 e T13 com o uso do isoxaflutole e T15, T19, T21 e T23 com o uso do flumioxazina. Estes ingredientes ativos (isoxaflutole e flumioxazina) apresentam tempo de meia vida médios de 30 dias, porém mecanismos de ação diferentes, um inibidor de carotenoides e outro Protox, respectivamente.

Ressalta-se que todos os tratamentos que apresentaram eficiência maior que 80\% no fim da avaliação (T3, T5, T7, T9, T11, T13, T15, T17, T19, T21, T23, T25 e T27) tiveram uma reaplicação do pré-emergente na linha de plantio aos 120 dias (operação de remonta).

A utilização de herbicidas pré-emergentes, com efeito residual prolongado, é um dos fatores que determinam grande eficiência no controle de plantas daninhas durante o período crítico de competição (Monquero et al., 2008).

Dentre os tratamentos com melhores eficiências, destacou-se estatisticamente o T3 (glyphosate + Isoxaflutole + remonta) por apresentar melhor controle aos 240 DAA, sendo que 
uma das características do herbicida isoxaflutole é a utilização em períodos de seca, devido ao seu alto poder de lixiviação e baixa sorção no solo. Em casos de alta precipitação, supõese que este produto não teria o mesmo residual como no período testado.

Recomenda-se também o uso do isoxaflutole para plantas alvo de folha estreita, que é uma das características da área estudada. Analisou-se também que o crescimento das mudas de eucalipto nos tratamentos que permaneceram com maior período de eficiência, acima de $70 \%$, apresentaram maior produtividade (T3, T5, T7, T13, T19 e T21), ou seja, seu crescimento inicial (arranque) não foi afetado, condições básicas para proporcionar fechamento da copa do plantio antes dos 365 dias de idade, reduzindo assim necessidade de operações adicionais de capina química e maior produtividade inicial.

Aos 240 DAA as plantas de E. grandis x E. urophylla estavam com as copas fechadas na linha de plantio, onde se encerrou a avaliação de eficiência.

\section{CONCLUSÃO}

- A remonta com pré-emergentes não provocou sintomas visuais de intoxicação no eucalipto;

- Todas as misturas de ingredientes ativos foram eficientes para o controle de plantas daninhas presentes na área.

- A remonta com os produtos pré-emergentes, 120 DAA, permitiu que a eficiência de controle permanecesse até o fechamento das copas do eucalipto, aos 240 dias após plantio;

- O tratamento T3 (glyphosate + isoxaflutole + remonta), apesar de apresentar pouca diferença estatística de outros tratamentos, apresentou excelente controle das plantas daninhas na linha e entrelinha aos 240 dias, sem intoxicação à cultura.

\section{AGRADECIMENTOS}

À Coordenação de Aperfeiçoamento de Pessoal de Nível Superior - Brasil (CAPES) e a Klabin S.A.

\section{REFERÊNCIAS}

Agostinetto, D., Tarouco, C. P., Markus, C., Oliveira, E., Silva, J. M. B. V., \& Tironi, S. P. (2010). Seletividade de genótipos de eucalipto a doses de herbicidas. Semina. Ciências Agrárias, Londrina, 31(3), 585-598. http://dx.doi.org/10.5433/1679-0359.2010v31n3p585.

Asociation Latinoamericana de Malezas - ALAM. (1974). Recomendaciones sobre unificación de los sistemas de evaluación en ensayos de control de malezas. ALAM, 1(1), 35-38.

Brasil. Ministério da Agricultura Pecuária e Abastecimento. (2019). Sistemas de Agrotóxicos Fitossanitários - AGROFIT. Disponível em: http://agrofit.agricultura.gov.br/agrofit_cons/principal_agrofit_cons. Retrieved in 2019, October.

Coimbra, J. L. M., Carvalho, F. I. F., \& Oliveira, A. C. (2004). Fundamentos do SAS aplicado à experimentação agrícola (246 p.). Pelotas: Edição Universitária, UFPel.

Costa, A. C. P. R., Costa, N. V., Pereira, M. R. R., \& Martins, D. (2012). Efeito da deriva simulada de glyphosate em diferentes partes da planta de Eucalyptus grandis. Semina: Ciências Agrárias, 33(5), 1663-1672. http://dx.doi.org/10.5433/1679-0359.2012v33n5p1663.

Dalazen, G., Kruse, N. D., Machado, S. L.O., Balbinot, A. (2015). Sinergismo na combinação de glyphosate e saflufenacil para o controle de buva. Pesquisa Agropecuária Tropical, 45(2), 249-256.

Indústria Brasileira de Árvores - IBÁ. (2018). Sumário Executivo. Brasília: IBÁ.

Kofler, I., \& Pandolfi, M. A. C. (2019). Condições de trabalho do trabalhador rural avaliando o impacto da exposição ao agrotóxico. Revista Interface Tecnológica, Taquaritinga, 16(1), 460-469.

Monquero, P. A., Binha, D. P., Silva, A. C., Silva, P. V., \& Amaral, L. R. (2008). Eficiência de herbicidas préemergentes após períodos de seca. Planta Daninha, 26(1), 185-193.

Pereira, F. C. M., \& Alves, P. L. C. A. N. (2015). Herbicides for weed control in eucalypt. Revista Brasileira de Herbicidas, 14(4), 333-347. http://dx.doi.org/10.7824/rbh.v14i4.425. 
Rezende, E. H. (2014). Aplicação de herbicidas pré-emergentes na implantação e reforma de áreas de Eucalyptus urophylla, antes e após o plantio (Dissertação de mestrado) Universidade Federal do Paraná, Curitiba. 91 p.

SAS Learning Edition. (2003). Getting started with the SAS Learning Edition (200 p.). Cary: SAS Learning Edition.

Sociedade Brasileira da Ciência das Plantas Daninhas - SBCPD. (1995). Procedimentos para instalação, avaliação e análise de experimentos com herbicidas (42 p.). Londrina: SBCPD.

Teixeira, J., Hakamada, R.E., Polli, H.Q., Bazani, J.H. (2015). Manejo de plantas daninhas através do uso de herbicidas em pré-emergência em área total com enfoque na mecanização. Série Técnica IPEF, 20(41), 47-58.

Tiburcio, R. A. S., Ferreira, F. A., Ferreira, L. R., Machado, M. S., Machado, A. F. L. (2012). Weed control and selectivity of flumioxazin in eucalyptus. Cerne, 18(4), 523-531. http://dx.doi.org/10.1590/S010477602012000400001.

Contribuição dos Autores: MS: Conceituação, Curadoria de Dados, Análise Formal, Investigação, Metodologia Administração do Projeto, Validação, Visualização, Escrita - Primeira Redação, Escrita - Revisão e Edição. MBC: Conceituação; Análise Formal, Obtenção de Financiamento, Investigação, Metodologia, Visualização, Recursos, Escrita - Primeira Redação; Escrita - Revisão e Edição. EDR: Visualização, Conceituação, Análise Formal, Investigação, Metodologia, Escrita - Primeira Redação; Escrita - Revisão e Edição. DTM: Visualização, Validação, Análise Formal, Escrita - Revisão e Edição. NJS: Conceituação; Obtenção de Financiamento, Análise Formal, Metodologia, Recursos, Visualização, Validação, Escrita - Revisão e Edição. 